\title{
The Effect of Film Morphology and Interface Structure on the Magnetic Properties of Fe-MgO Thin Films
}

\author{
S.R. Spurgeon*, S.E. Lofland**, A. Misra***, J.K. Baldwin***, and M.L. Taheri* \\ * Department of Materials Science \& Engineering, Drexel University, Philadelphia, PA \\ ** Department of Physics \& Astronomy, Rowan University, Glassboro, NJ \\ *** Center for Integrated Nanotechnologies, Los Alamos National Laboratory, Los Alamos, NM
}

The large magnetoresistance of Fe-MgO thin films makes them a promising candidate for use in faster, more efficient random access memory [1]. The magnetization, magnetocrystalline anisotropy, and magnetoresistance of these films all depend on the interfacial roughness and thickness of the Fe layer [2,3]. Additionally, a variation in deposition temperature by only a few hundred degrees can induce large changes in film morphology and magnetic behavior. It is therefore of great value to quantify the relationship between film morphology, interface structure, and the resulting magnetic properties of these composites.

Using electron beam evaporation we have deposited Fe films of varying thickness on $\mathrm{MgO}(001)$ films, at both 500 and $600{ }^{\circ} \mathrm{C}$. The films were subsequently capped with a nominal $10 \mathrm{~nm} \mathrm{Au}$ layer to prevent oxidation. These temperatures were chosen to yield both Fe islands $\left(500{ }^{\circ} \mathrm{C}\right)$ and uniform films $\left(600^{\circ} \mathrm{C}\right)$, to explore the effect of film uniformity on the composite's properties.

X-ray diffraction and reflectometry measurements were used to confirm the orientation, thickness, and structure of the films. Transmission electron microscopy (TEM) samples were then prepared using a focused ion beam (FIB) lift out technique, followed by low angle ion milling to remove damage and achieve electron transparency. High resolution imaging was conducted using a $200 \mathrm{kV}$ JEOL $2100 \mathrm{LaB}_{6}$ TEM. Magnetic hysteresis and anisotropy measurements were conducted using a Quantum Design MPMS SQUID magnetometer and a magneto-optical Kerr effect (MOKE) magnetometer.

Our results illustrate the complex effects of island structure and thickness on the magnetic properties of these composites. Changes in island connectivity and size with film thickness are clearly evident, as shown in Figure 1. It is possible to measure the quantity and type of defects present at the interface using high resolution TEM images (Fig. 2). We can relate interface structure to measured magnetic properties, which will eventually enable the precise design of these materials for novel magnetic memories.

References

[1] Slaughter, J. M. (2009). Annual Review of Materials Research, 39(1), 277-296.

[2] Salvador, C., et al. (2008). Journal of Physics D: Applied Physics, 41(20), 205005.

[3] Huang, Y., et al. (1993). Physical Review B, 47(1), 183-189. 


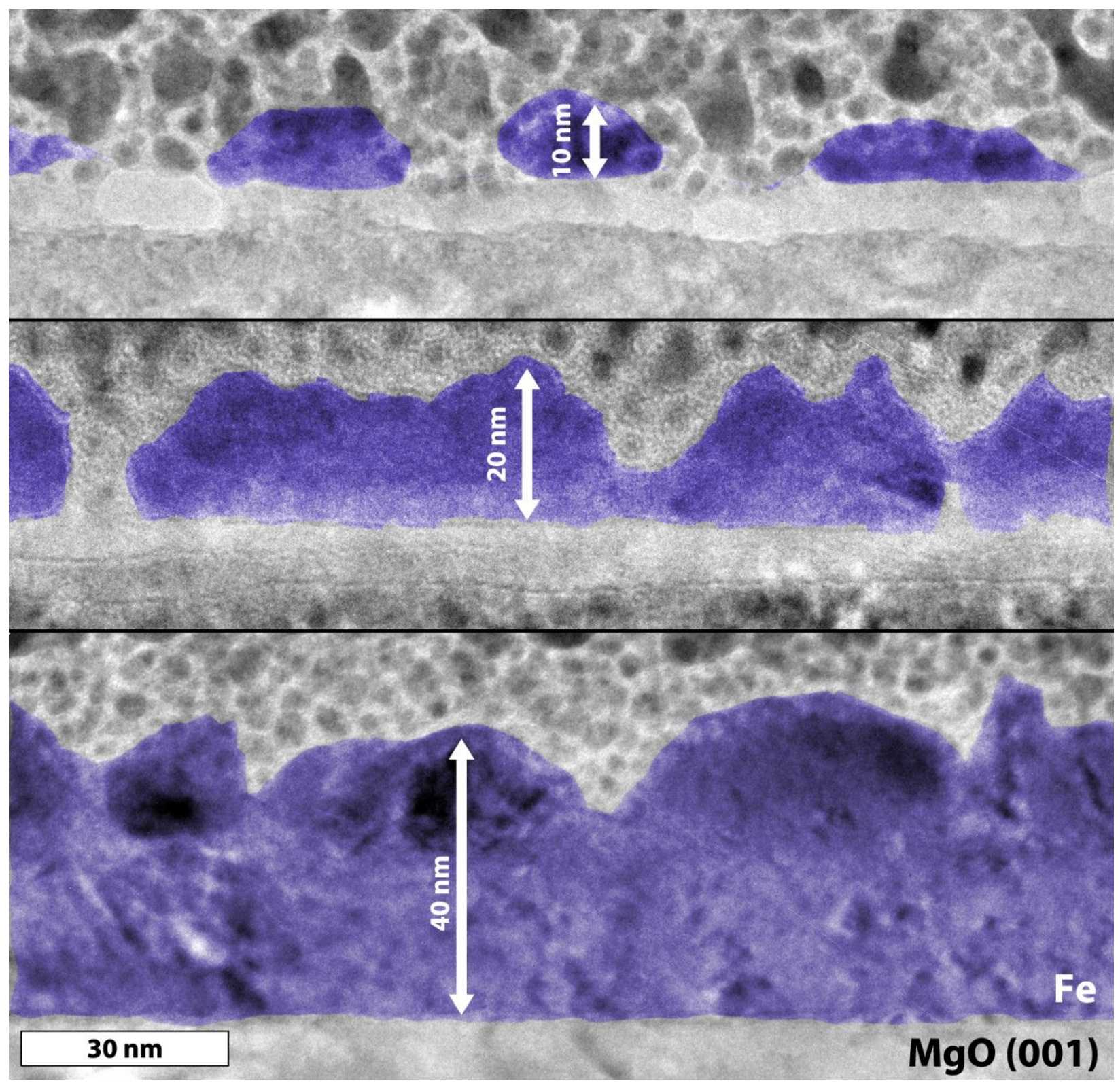

Figure 1: TEM montage showing the evolution of island morphology and connectivity with increasing film thickness. A color overlay has been added to guide the eye.

Figure 2: High resolution TEM image of the interface between a $\mathrm{Fe}$ island and the $\mathrm{MgO}(001)$ substrate.

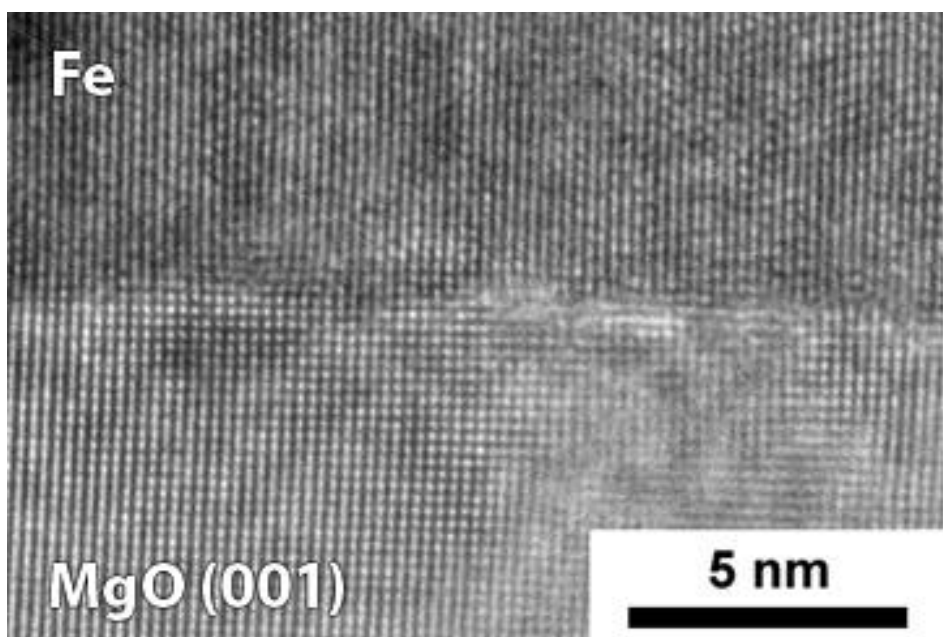

